\title{
Multi-sided metallization of textile fibres by using magnetron system with grounded cathode
}

\author{
RAFAŁCHODUN $^{1, *}$, BARTOSZ WICHER ${ }^{1}$, ŁUKASZ SKOWROŃSKI $^{2}$, \\ KatARZyna NOWAKOWSKA-LANGIER ${ }^{3}$, SEbastian OKRASA ${ }^{1}$, ANDrZEJ Grabowski ${ }^{2}$, \\ ROMAN MINIKAYEV ${ }^{4}$, KRZYSZTOF ZDUNEK $^{1}$ \\ ${ }^{1}$ Faculty of Materials Science, Warsaw University of Technology, Woloska 141, 02-507 Warsaw, Poland \\ ${ }^{2}$ Institute of Mathematics and Physics, UTP University of Science and Technology, \\ al. Prof. S. Kaliskiego 7, 85-796 Bydgoszcz, Poland \\ ${ }^{3}$ National Centre for Nuclear Research (NCBJ), A. Soltana 7, 05-400 Otwock, Poland \\ ${ }^{4}$ Institute of Physics, Polish Academy of Sciences, Lotnikow 32/46, 02-668 Warsaw, Poland
}

\begin{abstract}
The synthesis of coatings on textiles fibers enables functionalization of their properties e.g.: changing the reaction on IR radiation. In our experiment, a magnetron with a grounded cathode and positively biased anode was used as a source of plasma. A ring anode was positioned at $8 \mathrm{~cm}$ distance from the cathode. Samples of glass and cotton textile were placed at the plane of the anode. Ti and TiN coatings were deposited by sputtering of titanium target in $\mathrm{Ar}$ or $\mathrm{Ar}+\mathrm{N}_{2}$ atmosphere. SEM studies showed that, using the magnetron system described above, the textile fibers were covered by the $2 \mu \mathrm{m}$ to $3 \mu \mathrm{m}$ thick coatings. Unexpectedly, the coatings were deposited at both sides of the samples: the front side was exposed to glow discharge plasma and the backside was completely shaded from the plasma. IR optical investigation exhibited significant change in reflectance and transmittance of the coated textiles. The using of standard magnetron system (grounded anode and cathode at negative potential) resulted in a coating deposition at the textile side exposed to the plasma action only. We believe that the multi-sided deposition of coatings observed during the process run with magnetron with grounded cathode is a result of an ambipolar diffusion mechanism in the anodic potential drop region.
\end{abstract}

Keywords: magnetron sputtering; coatings deposition on textiles; optical properties

\section{Introduction}

It is obvious that textile materials are important and widely used for a variety of casual applications. The functionality of modern textiles could be adjusted to particular demands by changing their properties, especially surface properties such as: wear resistance, heat and electrical conduction, biocompatibility, antibacterial properties, antistatic properties and so on [1]. Conventional methods enhancing textiles surface properties are currently used for such purposes as: [2] printing, lamination [3], chemical plating [4], sonochemical reaction [2-5]. Recently, growing interest in Plasma Assisted Physical Vapor Deposition (PAPVD) for surface modification of textiles has been

*E-mail: rafal.chodun@inmat.pw.edu.pl observed. The magnetron sputtering method (MS) as the most widely used PAPVD method seemed to be a good choice [6-8]. Unfortunately, the most common problems for coatings deposition on textiles by MS are poor adhesion, cracking and limited thickness $[9,10]$. Also, it should be taken into account that, during MS process, coatings deposited on the surface of the textile side are exposed to plasma of glow discharge only and the shading phenomenon does not allow the plasma particles to penetrate deeply into the textile to cover fibers underneath the surface. This seems to be a significant disadvantage because deeper fibers could not be fully coated which could strongly affect the material properties. Lately, we have developed a specific plasma source - a magnetron in which the cathode is electrically grounded and a positively biased anode is separated from the magnetron body. Our 
latest study showed that despite the fact that the grounded magnetron cathode has the same potential as the grounded chamber body, the glow discharge plasma localizes on the surface of the magnetron cathode and a target sputtering occurs as in case of standard magnetron sputtering [11]. This modification leads to a stronger electric potential drop in the anode area in comparison to the standard magnetron because of the reversed relation of the magnetron electrode areas. We believe that the change of the electric potential at the anode as well as the electrode separation of the discharge electrodes could affect the plasma particles paths, especially in the anode zone. In our opinion, this could be used for more effective deposition of coatings on textiles by using the sputtering technique. In the presented experiment, the deposition effectiveness was studied for non-reactive as well reactive processes. As control parameters, the transmittance and reflectance of the uncoated and coated textiles surfaces were chosen.

\section{Experimental}

A diagram of the coating deposition system is shown in Fig. 1. The chamber is made of $40 \mathrm{~cm}$ diameter quartz glass pipe. The chamber is located vertically at the pump system inlet. The upper end of the chamber is closed by a stainless-steel lid. The lid is used as a mounting plate for the magnetron body, the anode holder and working gas supply. The lid can be both grounded and electrically isolated. The sputtering system consists of a self-made magnetron and the separated anode is placed $8 \mathrm{~cm}$ away from the cathode. The used magnetron magnetic system causes that the $\mathrm{B}_{Z 0}$ point is located at a distance of about $1 \mathrm{~cm}$ above the target surface. According to the Gencoa criterion [12], this means that our magnetron works as highly magnetically balanced. The solid state magnets are arranged in magnetron body to obtain the characteristic zone of sputtering in a form of a $25 \mathrm{~mm}$ to $30 \mathrm{~mm}$ diameter ring. The anode is made of an $8 \mathrm{~mm}$ copper pipe in the form of a $7 \mathrm{~cm}$ diameter ring. The magnetron body as well the anode holder can be electrically connected or isolated from the stainless steel lid. During the experiments, two magnetron electrodes arrangements were used: (a) the grounded cathode connected electrically with the grounded lid and positively biased anode and (b) the negatively biased cathode and the grounded anode connected with the grounded lid. This second arrangement made the magnetron system more alike to the typically used magnetron systems. As a sputtered material, a $48 \mathrm{~mm}$ diameter and $3 \mathrm{~mm}$ thick titanium target (Ti of grade 1) was used. The DPS [13, 14] power supply, operating at $100 \mathrm{kHz}$ frequency with $500 \mathrm{~Hz}$ modulation was used. Before the deposition process the $1200 \mathrm{~L} / \mathrm{s}$ turbomolecular pump evacuated the vacuum chamber to the base pressure of $5 \times 10^{-4} \mathrm{~Pa}$. The parameters of the coating deposition are shown in Table 1.

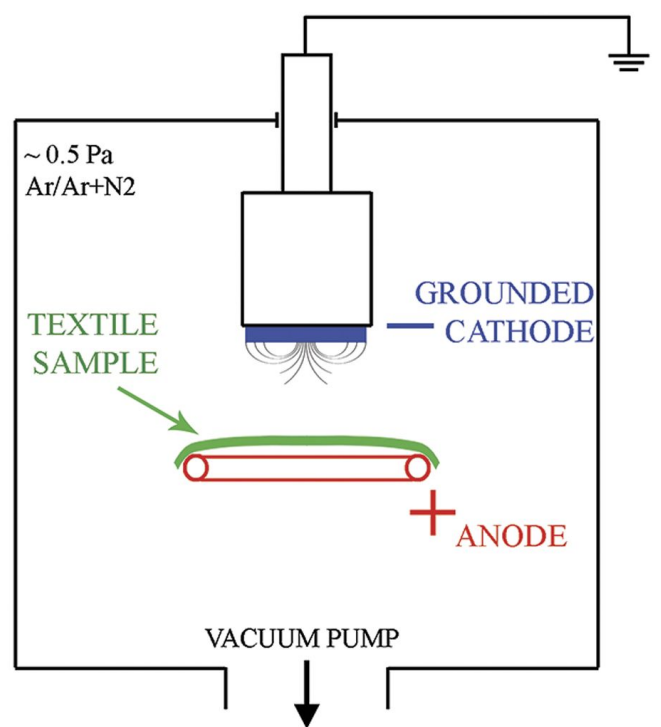

Fig. 1. Diagram illustrating the setup of process apparatus.

As test substrates for coatings composition and structural study, n-type ( $\left.\begin{array}{lll}1 & 0 & 0\end{array}\right)$ silicon wafers were used. The samples were placed perpendicularly to $\mathrm{Z}$ axis of the magnetron system, directly in the plane of the anode ring (Fig. 1). The structural characterizations of the layers were carried out by means of $\mathrm{X}$-ray diffraction using $\mathrm{CuK} \alpha$ radiation $(\lambda=1.54056 \AA)$. The morphology of structure was examined by means of ZEISS Ultra Plus. SEM images were taken at $45^{\circ}$. 
Table 1. Process parameters of coating deposition.

\begin{tabular}{|c|c|c|c|c|c|}
\hline \multirow[b]{2}{*}{ Substrate } & \multicolumn{2}{|c|}{ Ti coating } & \multicolumn{3}{|c|}{ TiN coating } \\
\hline & Glass textile & Cotton textile & Silicon & Glass textile & Cotton textile \\
\hline Ar partial pressure $[\mathrm{Pa}]$ & 0.45 & 0.45 & 0.45 & 0.45 & 0.45 \\
\hline $\mathrm{N}_{2}$ partial pressure $[\mathrm{Pa}]$ & - & - & 0.05 & 0.05 & 0.05 \\
\hline Power $[\mathrm{kW}]$ & 3 & 2 & 3 & 3 & 2 \\
\hline Time [min] & 60 & 10 & 10 & 30 & 10 \\
\hline
\end{tabular}

In further experiment we used circular samples of textiles (glass and cotton) as substrates for coating deposition. The samples were placed at the same location as the silicon ones. During the deposition, only one side of the textiles sample was exposed to the plasma emitted from the Ti target.

The Ti and TiN coatings were investigated by means of SEM. Examined samples were taken by cutting off $1 \times 1 \mathrm{~cm}$ scratch of textile, then the surface and cut edges were observed to find out if the fibers had been fully covered. For this study, the ZEISS Ultra Plus SEM was used.

Reflectance and transmittance spectra were measured in the spectral range of $200 \mathrm{~nm}$ to $2500 \mathrm{~nm}$ by means of the Cary 5000 spectrophotometer (from Agilent) equipped with an integrating sphere (both specularly and diffuse reflected beams were recorded). The data were corrected using the standard baseline correction procedure.

\section{Results and discussion}

In our previous work we suggested that localization of glow discharge plasma at grounded surface is possible by use of locally applied magnetic field [11]. Plasma localization occurs because the breakdown voltage needed to maintain the glow discharge is lower for magnetized ambient than for the unmagnetized one [15-17]. We believed that the sputtering would be limited to the region where magnetic field is applied, avoiding sputtering of other elements at the ground potential. To confirm this, we studied XRD spectra of TiN coating deposited on silicon substrate. The spectrum presented in Fig. 2 consists of the peaks assigned to $\mathrm{TiN}$ and $\mathrm{Si}$ planes only. No extra phases are observed. TiN does not show any preferred orientation of growth. Obtained lattice parameters have been plotted against the Nelson-Riley function. Extrapolation of the function gave true value of lattice parameter, $a_{o}=4.242 \AA$. This value is very close to that reported in literature (JCPDS Card No. 381420) and suggests that obtained TiN phase is stoichiometric. In our opinion, it proves that the sputtering is limited to the surface of the target material. Another evidence of localization the sputtering zone is an image in Fig. 3 where the target sputtering trace is presented.

Fig. 4 presents the images of glass textiles samples after deposition processes carried out with the use of a magnetron working with the grounded cathode. Despite the fact that during the deposition, only one side of the samples was exposed to the plasma source, both sides seem to be covered well by the Ti and TiN coatings. Nevertheless, the color difference between both sides of the samples suggests that the coating at the sample backside seems to be slightly thinner than the coating on the plasma exposed side. For comparison, we present in the first row of Fig. 4 the result of a deposition process carried out with the electrode setup system alike to the standard magnetron sputtering arrangement. In this case, the sample front side (the side exposed to the plasma) was covered by the Ti coating while the sample backside stayed white and uncovered. Fig. 4 shows a basic difference between both versions of the MS method. In case of sputtering carried out with the use of the grounded cathode, particles penetrated the textiles and textiles fibers which were covered omnidirectionally. This contrasts with the standard magnetron sputtering, where the coatings are deposited only on the exposed side of the textile. It is worth noting 

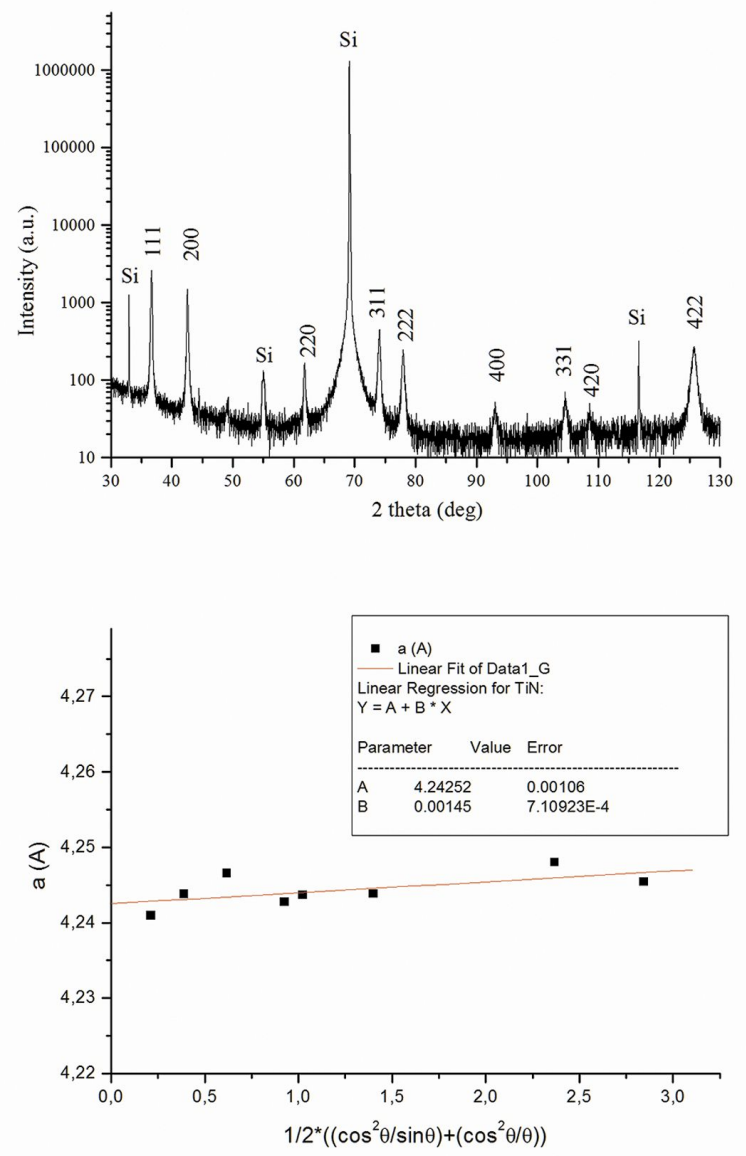

Fig. 2. XRD spectrum of TiN coating deposited on silicon substrate (top), extrapolation of lattice parameters calculations (bottom).

that the one-sided covering during the coatings deposition on textiles by the standard magnetron sputtering is typical and commonly experimentally observed. For example, deposition processes for coatings on fibers by MS method were studied by Ziaja et al. [18, 19]. They studied the effectiveness of coatings deposition on non-woven fabrics used for shields of electromagnetic radiation applications. The cited works clearly reported that metallization of fabrics by MS method occurred only at the exposed side of textile. Additionally, the authors confirmed by resistance measurements that the exposed surface of textile was not covered completely.

Fig. 5 shows SEM images of glass fibers taken from both sides of the glass textiles sample

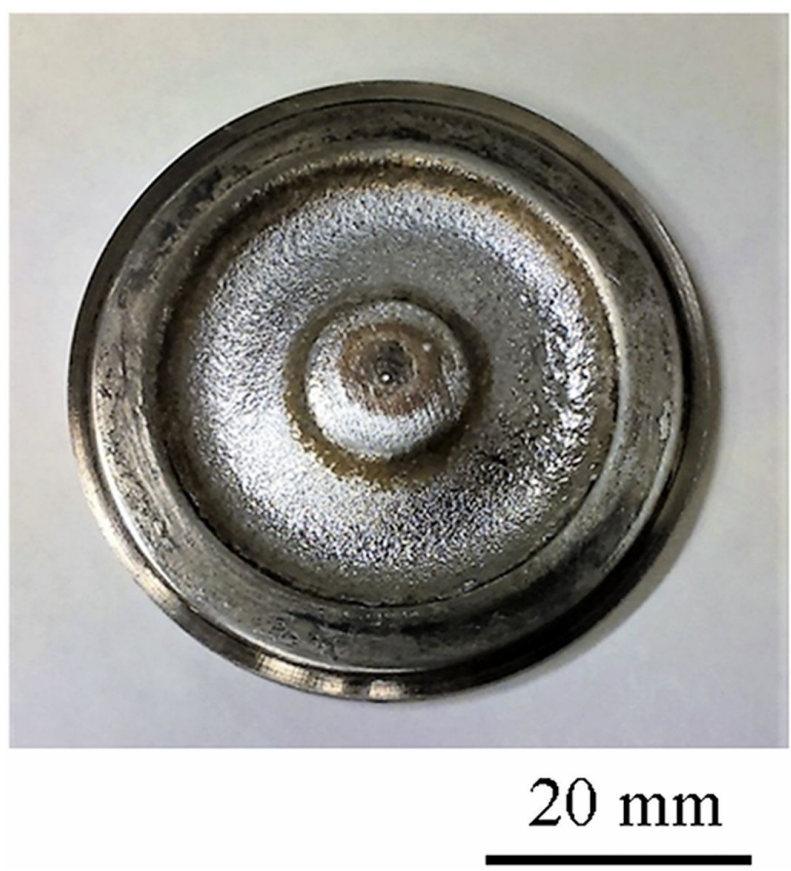

Fig. 3. Ti target after $50 \mathrm{~h}$ of sputtering.

compared with uncoated ones. Additionally, SEM images of coatings deposited on silicon substrates in a reference process are presented. Images were taken randomly from the surface of the textiles at the cut edges of fibers. The textile fibers at the exposed side are fully covered. The coatings are $\sim 2 \mu \mathrm{m}$ thick both for the Ti and TiN coatings. Evidences of delamination and cracking are observed only in the zones where the fibers were mechanically cut. Untouched fragments of the fibers appeared as crack-free. Previous studies concerning deposition of coatings on textile fibers by the magnetron sputtering method reported flaws such as cracking and adhesion faults. Wei et al. [9] observed that the tendency to delamination of coatings comes from an insufficient energy available for bonding the coating with fibers. Additionally, the authors pointed out that cracking mechanism appeared for copper coatings on polypropylene fibers thicker than $50 \mathrm{~nm}$. A similar conclusion was presented by $\mathrm{Xu}$ et al. [10] concerning $\mathrm{TiO}_{2}$ coatings on polyester fibers. Poor adhesion can be also explained as a result of insufficient activation of fibers surface, internal stress, and differences in the thermal expansion coefficient. Chen et al. [20, 21] improved the adhesion of metallic coatings to PET 

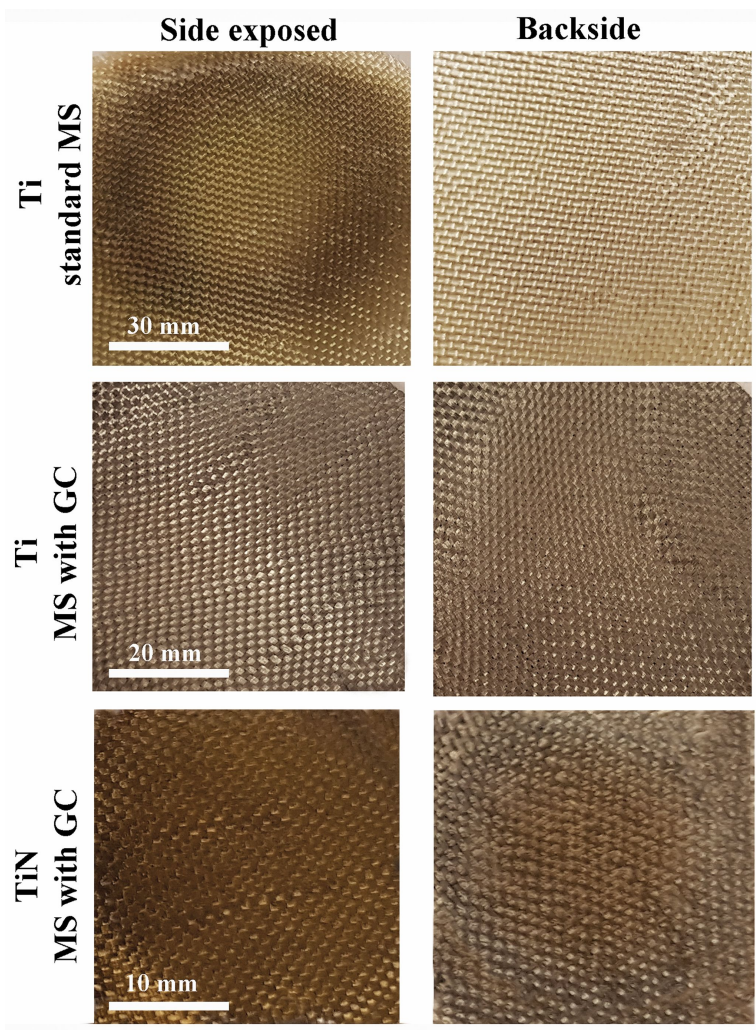

Fig. 4. Images of textiles surfaces after deposition processes. Images present both sides of samples: exposed to plasma source (left column) and shaded from plasma (right column).

fibers by using the HiPIMS method instead of the standard MS method. This high-power modification of the magnetron sputtering method characterized by a higher power density and higher ionization state of plasma enabled the production of more energetic plasma [22].

In proposed by us MS version, we assume that using a grounded cathode results in a significant modification of the plasma distribution and plasma energy in the anode zone where the textiles are placed. One should consider that in this arrangement the cathode surface area is much larger than the anode surface area. The cathode surface area consists of the area of grounded magnetron cathode ("magnetized cathode") and the area of grounded lid ("non-magnetized cathode"). The grounded lid acts electrically in the system as the cathode despite the fact that plasma is located only at the magnetron

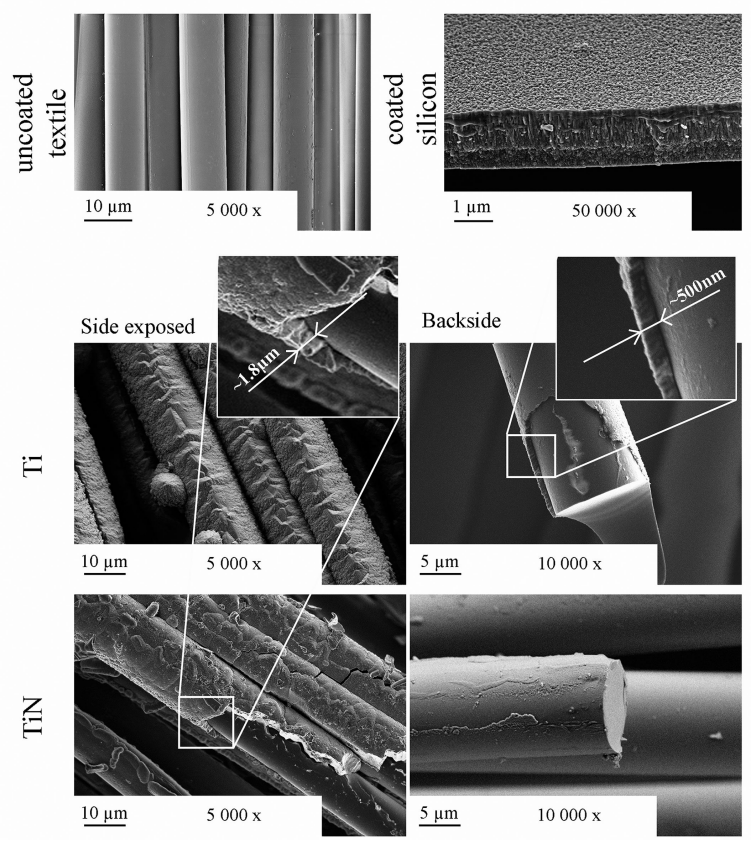

Fig. 5. SEM images of: uncoated textile fibers (top left), test silicon substrate coated by TiN (top right) and images of textiles surface after deposition processes. SEM images present both sides of samples: exposed to plasma source (left column) and shaded from plasma source (right column).

cathode. The total surface area of the grounded cathodes is much larger than the surface area of the anode, which results in a high electric field at the anode potential drop. This anode potential drop strongly affects the electrons of plasma of glow discharge, accelerating them to the anode. Because of the ambipolar diffusion [23, 24] and the interparticle collisions in the plasma, heavier plasma particles are also accelerated in the direction of the anode. The most energetic particles can pass the anode ring and, if charged, undergo further acceleration by the positive electric field. It seems, provided the textiles sample was placed at the center of the anode area, that the energetic plasma particles could easily penetrate the textiles structure covering their fibers and also approach the textile backside not exposed directly to the plasma emitted from the target area. During the standard MS process, the anode potential drop is lower than in case of the process with cathode at grounded potential because of the inferior ratio of the electrode 

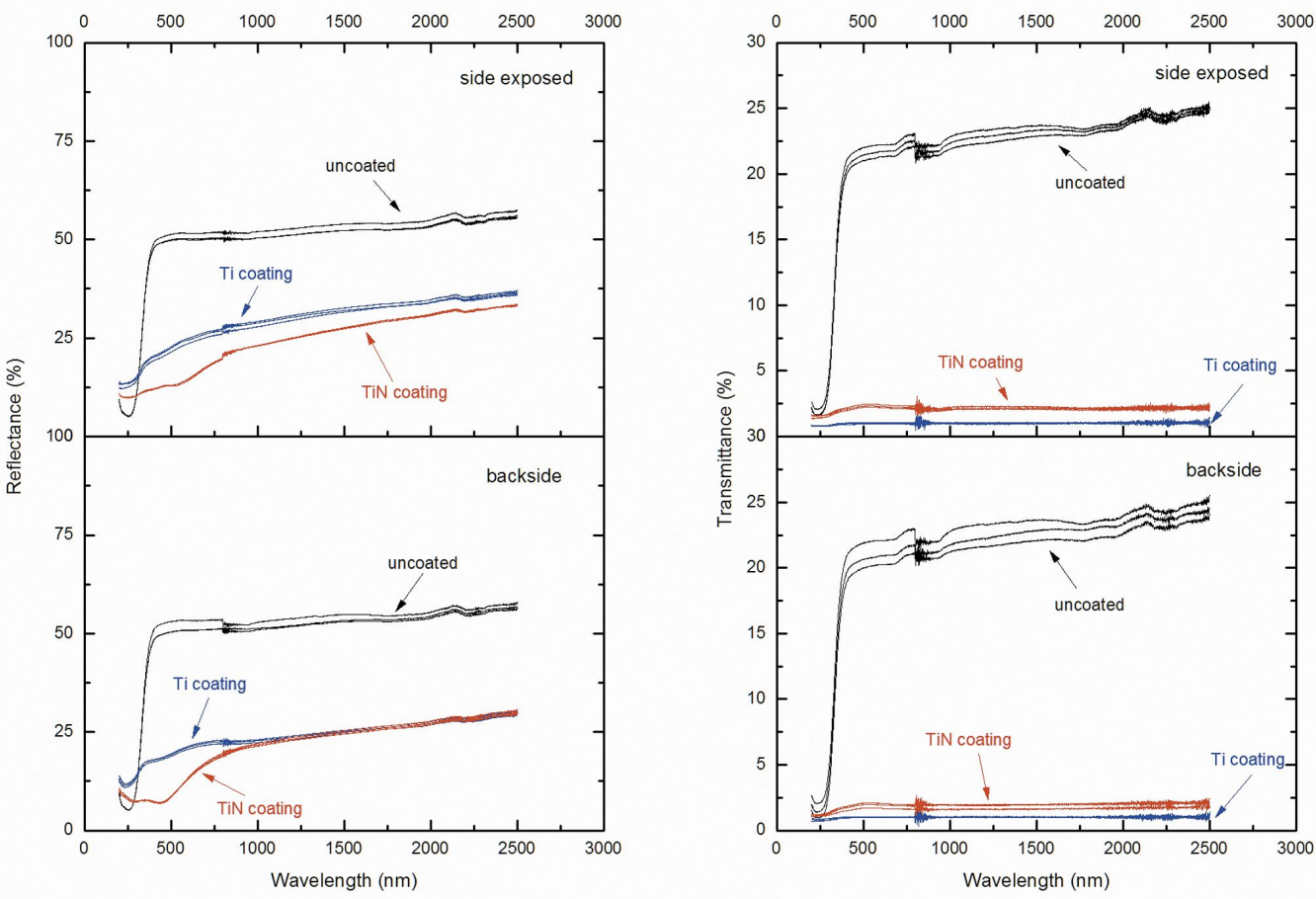

Fig. 6. Reflectance and transmittance spectra taken from both sides of glass textiles.

surface areas as well as the fact that the lid has the same electric potential as the grounded anode.

One of the most obvious properties of the modified textile seems to be its reaction to the IR radiation. To find out how the covering of the textiles influences their optical properties, the optical transmission and reflectance examinations were carried out up to $2500 \mathrm{~nm}$ radiation. Fig. 6 presents the results of reflectance and transmittance measurements of glass textiles. Both sides of the samples were investigated. Reflectance and transmittance were reduced after deposition of the coatings. Furthermore, there was no significant difference between both sides of the textiles, even if we proved that coatings on opposite sides of the samples were characterized by different thicknesses. The titanium coating appeared more reflective and less transmitting than titanium nitride. The character of the obtained spectra suggests that glass textile covered by $\mathrm{Ti}$ and $\mathrm{TiN}$ coatings not only reduce transmission but also scatter the electromagnetic radiation which is very promising for their potential applications.
Encouraged by the positive results obtained for glass textiles, we decided to synthesize coatings on cotton textiles. Taking into consideration much lower thermal stability of cotton fibers than those of glass, we reduced the power and length of the deposition process (Table 1). The character of cotton transmittance and reflectance spectra is more complex which probably results from properties of fabrics we used in this experiment. Nevertheless, the positive reaction of coated textiles to EM radiation is also observed. The spectra have been shown in Fig. 7.

\section{Conclusions}

When studying the possibility to cover textiles by the metallic coatings for increasing their useful properties for the human protection against heat we paid attention to the special arrangement of magnetron electrodes. We decided to use the grounded cathode arrangement of balanced magnetron and a separately located positively biased anode made in the form of a ring. We assumed that in such unusual 

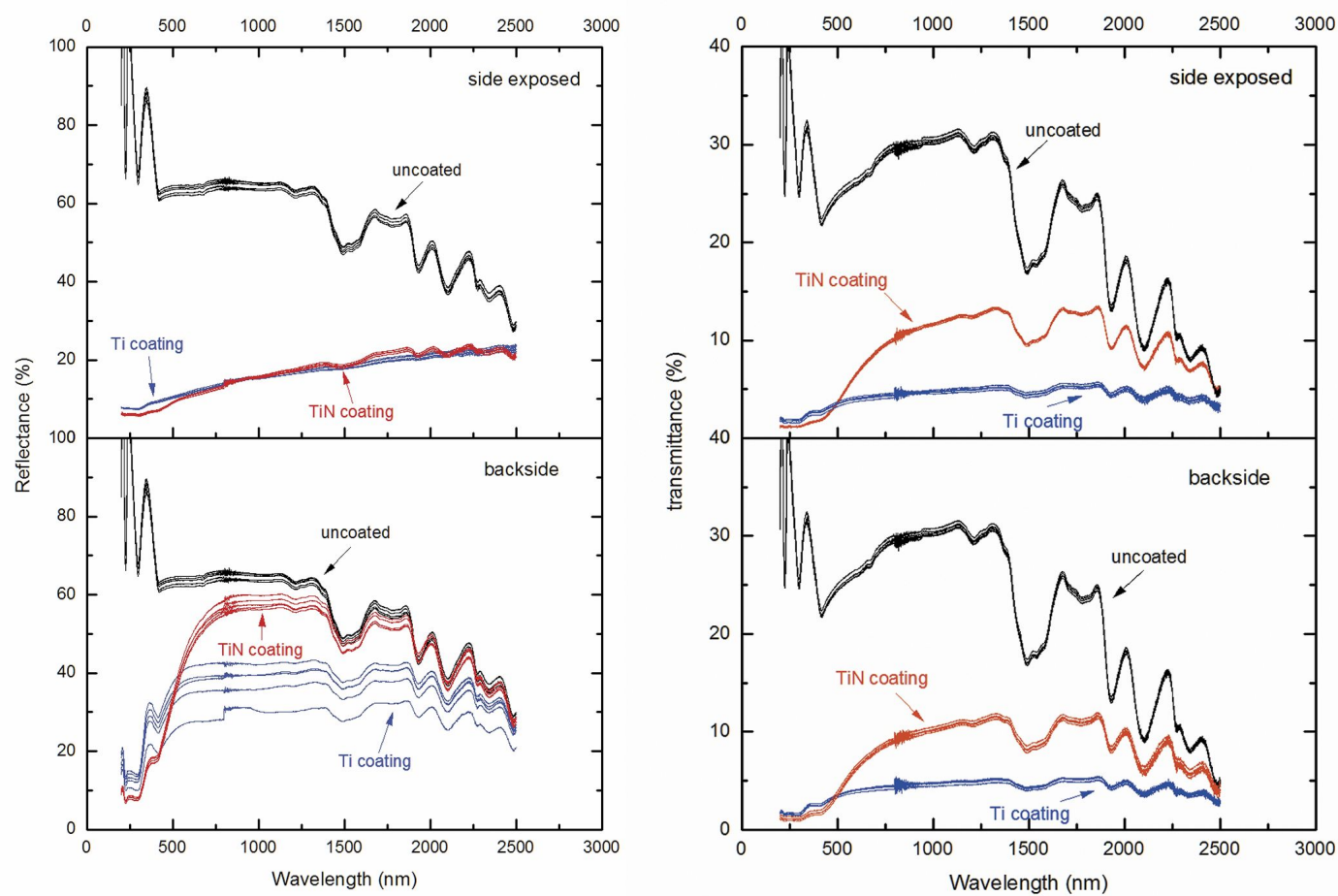

Fig. 7. Reflectance and transmittance spectra taken from both sides of cotton textiles.

magnetron setup the ambipolar diffusion effect enhanced by the strong anode potential drop could be especially beneficial for the deposition of coatings on textiles. Our experiments proved our assumptions showing a multi-sided covering effect for the textiles samples located directly on the ring-shaped anode. According to our best knowledge, such a multi-side covering effect during the modification of textile surface by plasma method has been observed for the first time. It seems to us that the experiment arrangement could be easily adapted to the regular massive production of coatings on textiles on an industrial scale.

\section{Acknowledgements}

This work was supported by the National Science Centre within the Project No. 2013/09/B/ST8/02418.

\section{References}

[1] KoncAR V., Smart Textiles and Their Applications, Woodhead Publishing, 2016.

[2] Robert S., Athey J.R., Coating Technologies Handbook, 91 (2001), 687.

[3] MCCARTY C., MCQuAID M., Structure and Surface: Contemporary Japanese Textile, Museum of Modern Art, New York, 1998.
[4] Jiang S., Newton E., Yuen C., Kan C., Text. Res. $J ., 76$ (2006), 57.

[5] Raja A., Thilagavathi G., Kannaian T., Indian J. Fibre Text. Res., 35 (2010), 59.

[6] Scholz J., Nocke G., Hollstein F., WeissBACH A., Surf. Coat. Technol., 192 (2005), 252.

[7] Depla D., Segers S., Leroy W., VAN Hove T., VAN PARYs M., Text. Res. J., 81 (2011), 1808.

[8] Yip J., Jiang S., Wong C., Surf. Coat. Technol., 204 (2009), 380.

[9] Wei Q., Xiao X., Hou D., Surf. Coat. Technol., 202 (2008), 2535.

[10] Xu Y., Wang H., Wei Q., LiU H., Deng B., J. Coat. Technol. Res., 7 (2010), 637.

[11] ChOdun R., NOWAKOWSKA-LANGIER K., ZDUNEK K., OKRASA S., Nukleonika, 61 (2016), 191.

[12] Gencoa Ltd., www.gencoa.com/balance_and_ unbalance accessed in 2016.

[13] Posadowski W.M., Wiatrowski A., Dora J., RAdZIMski Z.J., Thin Solid Films, 516 (2008), 4478.

[14] Dora J., Polish Patent No. 313150, 1996.

[15] Petraconi G., Maciel H. S., Pessoa R. S., MuRAKAMI G., MASSi M., OtANi C., URUChi W.M.I., Sismanoglu B.N., Braz. J. Phys., 34 (4b) (2004), 1662.

[16] Ledernez L., Olcaytug F., Urban G., Contrib. Plasma Phys., 52 (4) (2012), 283. 
[17] Nunez Y., Wemans A., Gordo P. R., Vacuum, 81 $(11-12)(2007), 1511$.

[18] Ziaja J., OzimeK M., Koprowska J., EMC Europe 2009 Workshop, (2009), 1.

[19] Ziaja J., Koprowska J., Janukiewicz J., Fiber. Text. East. Eur., 16 (2008), 64.

[20] Chen Y., Chin Hsu C, Liang He J., Surf. Coat. Technol., 232 (2013), 868.

[21] Chen Y., Wu G., Liang He J., Mater. Sci. Eng. C, 48 (2015), 41.
[22] Lundin D., LARsson P., Wallin E., LATtemann M., Brenning N., Helmersson U., Plasma Sources Sci. Technol., 17 (2008), 035021.

[23] RAIZER Y.P., Gas Discharge Physics, Springer, Berlin, 1991.

[24] Lieberman M.A., Lichtenberg A.J., Principles of Plasma Discharges and Materials Processing, Wiley, Hoboken, 2005.

Received 2017-03-13

Accepted 2017-09-04 